\title{
O VALOR DA TOMOGRAFIA COMPUTADORIZADA HELICOIDAL SEM CONTRASTE NA AVALIAÇÃO DE PACIENTES COM DOR NO FLANCO*
}

\author{
Mário de Melo Galvão Filho1, Giuseppe D'Ippolito1, Luiz Guilherme Hartmann², Charles Rosenblatt \\ Arcílio Jesus Roque ${ }^{3}$, José Alaor Figueiredo ${ }^{3}$, Maria Lúcia Borri ${ }^{1}$, Angela Borri Wolosker ${ }^{1}$
}

Resumo Os nossos objetivos foram estabelecer a eficácia da tomografia computadorizada sem contraste em diagnosticar a ureterolitíase, calcular a freqüência dos principais sinais tomográficos e medir a concordância interobservador, comparando-a com os resultados obtidos na urografia excretora, realizando estudo prospectivo duplo-cego em 25 pacientes com cólica nefrética. Em ambos os procedimentos avaliamos a existência de aumento renal, hidronefrose e cálculo ureteral. Deste último, descrevemos a sua localização e dimensão. Nos exames de tomografia computadorizada sem contraste procuramos também por estrias perirrenais, edema periureteral e o sinal do halo. Em 23 dos 25 exames de tomografia computadorizada sem contraste (92\%) e em 17 das 25 urografias excretoras $(68 \%)$ houve concordância dos resultados entre os dois observadores. Em 21 dos 25 pacientes os resultados dos dois métodos foram concordantes. Concluímos que a tomografia computadorizada sem contraste apresenta eficácia superponível à urografia excretora na avaliação de pacientes com cólica nefrética, sendo um método reprodutível e sem promover o desconforto do uso do contraste endovenoso.

Unitermos: Litíase. Ureter. Rim. Tomografia computadorizada.

Abstract The value of unenhanced helical computed tomography in the evaluation of patients with pain in the flank. Our purpose was to evaluate the accuracy of unenhanced helical computed tomography in the diagnosis of ureteral lithiasis, to establish the frequency of the main computed tomography findings, and to assess and compare the interobserver agreement with the results of conventional intravenous urography. A prospective double-blind study was carried out in $\mathbf{2 5}$ patients referred for unenhanced helical computed tomography and conventional intravenous urography due to nephretic colic. Both methods were employed to evaluate the size of the kidneys, hydronephrosis and the size and location of ureteral stones. Perirenal hyperdensities, periureteral edema and the halo sign were also assessed by unenhanced helical computed tomography. In 23 of the 25 unenhanced helical computed tomography examinations (92\%) and in 17 of the 25 conventional intravenous urography examinations (68\%) there was agreement on the results of the two observers. In 21 of the 25 patients, there was agreement between the results of both methods. We concluded that unenhanced helical computed tomography is as efficient as conventional intravenous urography in the evaluation of patients with nephretic colic, is a reproducible method, and avoids the discomfort of the use of intravenous contrast.

Key words: Lithiasis. Ureter. Kidney. Computed tomography.

\section{INTRODUÇÃO}

A cólica nefrética representa a principal queixa dos pacientes com cálculo nas vias urinárias ${ }^{(1)}$ e apresenta maior incidência entre os adultos (40-60 anos) do sexo masculino ${ }^{(2)}$. Os cálculos podem

* Trabalho realizado no Serviço de Tomografia Computadorizada do Complexo Hospitalar São Luiz, São Paulo,

SP.

1. Doutores em Radiologia e Médicos do Serviço de Tomografia Computadorizada e Ressonância Magnética do Complexo Hospitalar São Luiz e do Departamento de Diagnóstico por Imagem da Escola Paulista de Medicina.

2. Radiologista do Complexo Hospitalar São Luiz e do Departamento de Diagnóstico por Imagem da Escola Paulista de Medicina.

3. Urologistas do Complexo Hospitalar São Luiz.

Endereço para correspondência: Dr. Mário de Melo Galvão Filho. Avenida Jacutinga, 255, $5^{\circ}$ andar, Moema. São Paulo, SP, 04515-030. E-mail: mgalvaof@uol.com.br

Aceito para publicação em 14/2/2000. ocorrer em qualquer parte do trato urinário, sendo o rim o local mais freqüente, seguido pelo ureter e bexiga, respectivamente $^{(3)}$.

A urografia excretora (UGE) apresentava, até um passado próximo, a melhor eficácia entre os exames de diagnóstico por imagem. No entanto, é um método que prescinde da utilização do meio de contraste iodado e que apresenta potencial alérgico não-desprezível ${ }^{(4-6)}$.

Nos últimos anos, a tomografia computadorizada helicoidal sem contraste (TCSC) oral ou intravenoso tem despontado como alternativa útil, com elevada sensibilidade e especificidade no diagnóstico da cólica nefrética ${ }^{(7)}$.

Os nossos objetivos foram estabelecer a eficácia da TCSC em diagnosticar a ureterolitíase, a freqüência dos principais sinais tomográficos e medir a concordância interobservador, comparandoa com os resultados obtidos com a UGE.

\section{MATERIAL E MÉTODOS}

Realizamos estudo prospectivo duplo-cego em 25 pacientes que procuraram o serviço de urgência do Complexo Hospitalar São Luiz com queixa de cólica nefrética e que foram submetidos a exames de TCSC e UGE num intervalo de até duas horas. Destes 25 pacientes, 15 eram do sexo feminino e dez, do sexo masculino, com idade variando entre 18 e 62 anos (média de 40 anos).

Os exames de TCSC foram realizados em um aparelho helicoidal (Helicat II, 
Marconi) utilizando-se técnica espiral. Os exames iniciavam-se com um topograma abdômino-pélvico, onde eram marcados cortes desde o pólo superior do rim mais alto até a sínfise púbica. A utilização de "pitch" de 2,0 permitiu a aquisição de toda a extensão demarcada, em uma única apnéia. A espessura dos cortes utilizada foi de $6,5 \mathrm{~mm}$ e reconstruções a cada 3,0 mm. Não foram utilizados contrastes oral ou endovenoso. Os exames de UGE foram realizados por meio da injeção endovenosa de 50-100 ml de contraste iodado iônico ou não-iônico, em função do peso e antecedentes alérgicos do paciente. Após a injeção, foram realizados cortes planigráficos, incidências oblíquas, ortostáticas e radiografias de retardo, quando necessário.

Os exames foram analisados por dois radiologistas com experiências diferentes. Um deles, com oito anos de prática (observador A), e o outro, com três anos de prática (observador B). Quando havia discordância entre os examinadores, um terceiro radiologista, mais experiente, avaliava os exames, para estabelecer o resultado que seria utilizado no cálculo da eficácia dos métodos. É importante salientar que os exames de TCSC e UGE foram analisados com um intervalo de 14-28 dias e que as TCSCs eram avaliadas primeiro. Procuramos, desta maneira, evitar influências e memorizações que pudessem falsear os resultados.
Os três radiologistas estudaram e discutiram previamente os sinais a serem observados, para que não houvesse discrepâncias nas análises. Na TCSC e na UGE foi avaliada a existência de aumento renal, hidronefrose e cálculo ureteral. Deste último, descreviam a sua localização (junção ureteropiélica, ureter médio, ureter distal e na junção ureterovesical) e dimensão (diâmetro maior ou menor que $5 \mathrm{~mm}$ ). Nos exames de TCSC procuraram também por estrias perirrenais, edema periureteral e o sinal do halo, que permitiria diferenciar cálculo ureteral de flebólito.

A reprodutibilidade dos métodos, concordância interobservador e eficácia global entre os métodos foi calculada pelo teste de $\mathrm{McNemar}\left(\mathrm{p}>0,05^{*}\right)$. Para a nossa amostra, o teste de McNemar não foi aplicável para discordâncias em número inferior a cinco.

\section{RESULTADOS}

\section{Aumento renal}

Ambos os observadores identificaram o aumento renal (Figura 1) em 20 (80\%) dos pacientes e também a sua ausência em quatro (16\%) dos 25 pacientes. Em apenas um paciente houve discordância (Tabela 1). Apesar do teste de McNemar não ser aplicável nesta situação, é evidente que existe elevada concordância entre os observadores. Em 24 (96\%) dos
25 pacientes houve a mesma avaliação (Tabela 1).

\section{Hidronefrose}

Os examinadores A e B observaram hidronefrose (Figura 2) em 19 (76\%) dos 25 pacientes. Os observadores não concordaram em seis (24\%) dos 25 pacientes (Tabela 1). A análise estatística demonstra que não houve discordância significativa entre os observadores.

\section{Sinal do halo}

O sinal do halo se caracteriza por espessamento parietal do ureter, visto como densidade de partes moles, circular, ao redor de um cálculo ureteral (Figura 3). Em 18 (72\%) dos 25 exames de TCSC os observadores A e B concordaram quanto à presença do sinal do halo (Tabela 1). O teste de McNemar demonstrou que não existe discordância significativa entre os observadores.

\section{Estrias perirrenais}

As estrias perirrenais são hiperdensidades radiadas localizadas no espaço perirrenal (Figura 4) e que podem traduzir dificuldade no esvaziamento do sistema coletor. Foram verificadas em 18 (72\%) pacientes e em dois $(8 \%)$ não foram vistas por ambos os radiologistas (Tabela 1). O teste estatístico também determinou a concordância entre os observadores.

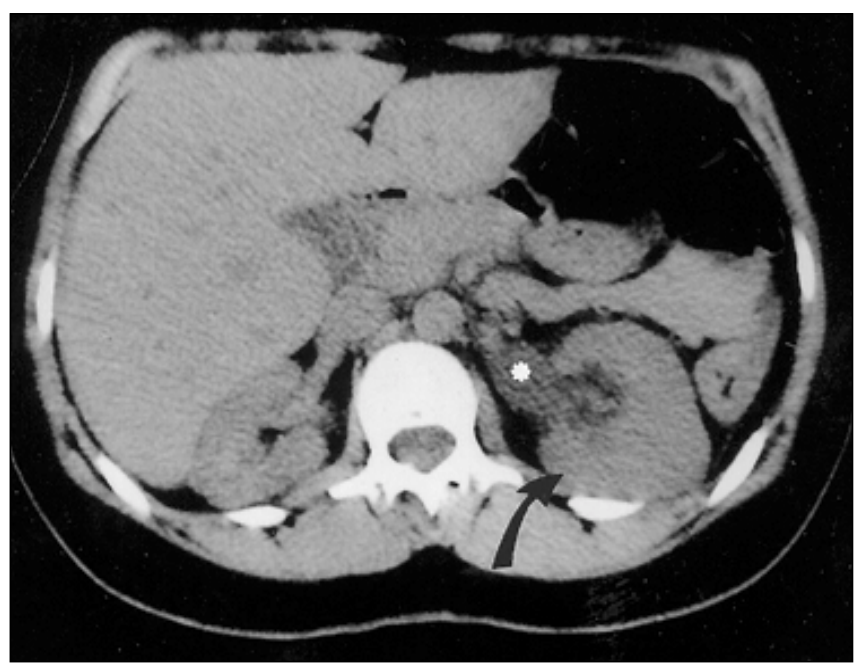

Figura 1. TCSC evidenciando importante aumento renal esquerdo (seta), comparado ao rim direito e associado a hidronefrose (asterisco).

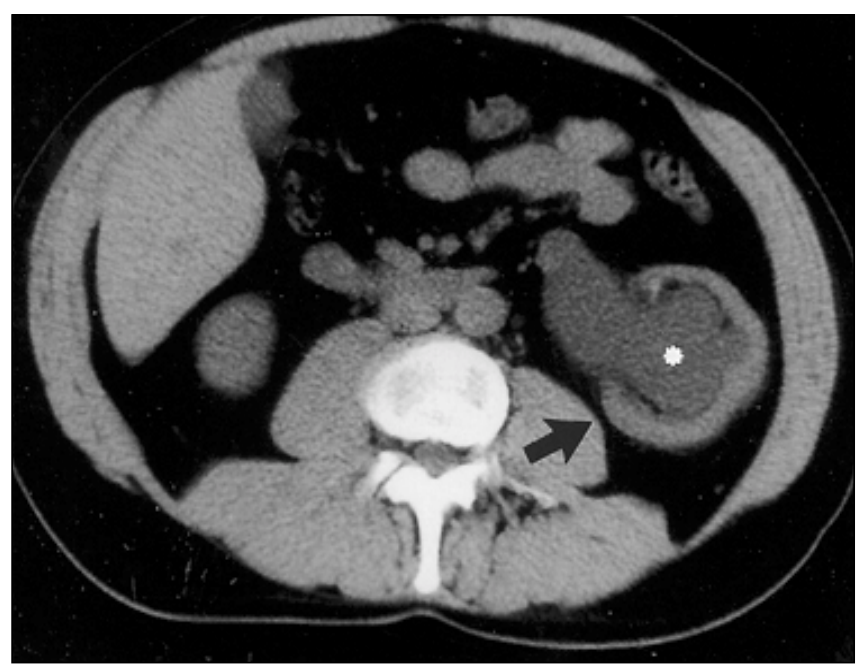

Figura 2. TCSC demonstrando aumento renal esquerdo (seta) e volumosa hidronefrose (asterisco) associada a redução do parênquima, em paciente com cólica à esquerda e irradiação para testículo. 


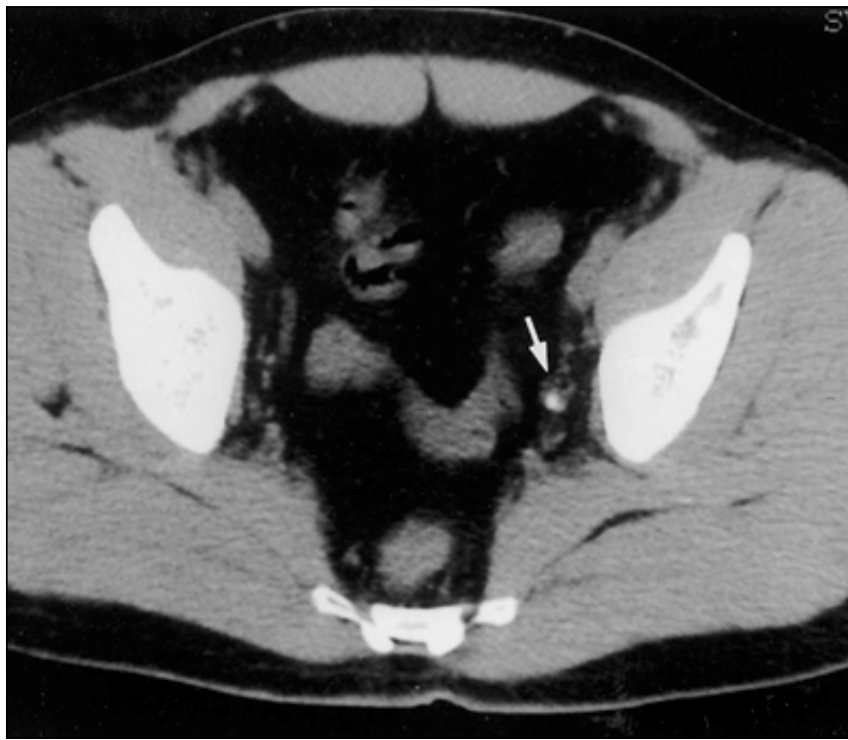

Figura 3. TCSC na altura da pelve demonstrando cálculo ureteral esquerdo com edema parietal (sinal do halo) e estrias periureterais (seta).

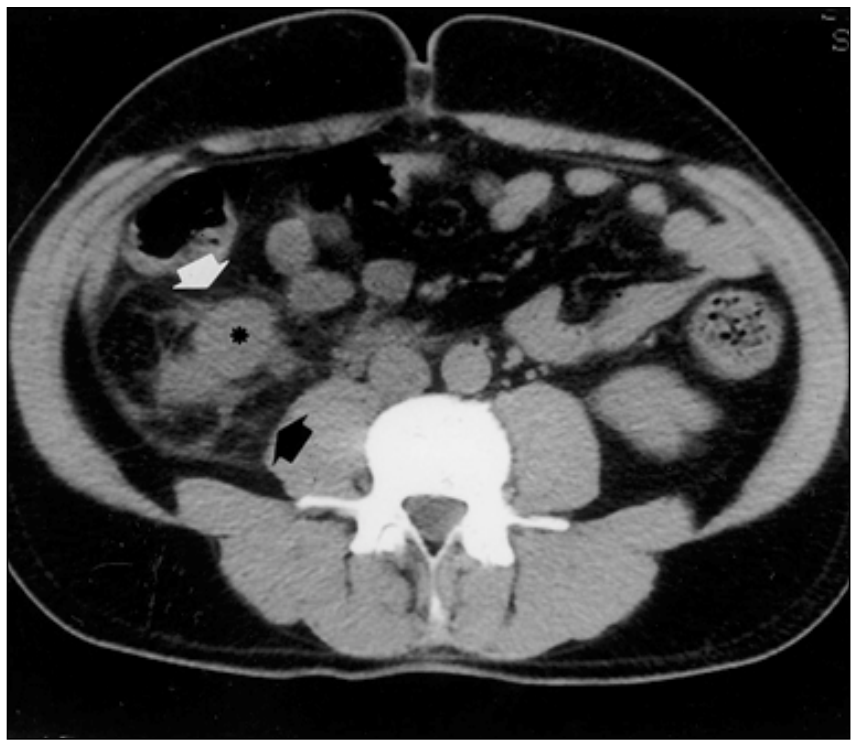

Figura 4. TCSC mostrando, junto ao pólo inferior do rim direito (asterisco), estrias hiperdensas (setas) que caracterizam indiretamente uma obstrução a montante.

\section{Estrias periureterais}

As estrias periureterais (Figura 3), assim como as estrias perirrenais, são hiperdensidades radiadas ao redor do ureter. Estavam presentes em 13/25 (52\%) dos pacientes e ausentes em quatro (16\%), segundo os dois observadores. Em oito pacientes não houve concordância dos resultados (Tabela 1). Apesar da discordância identificada, ela não foi considerada estatisticamente significante pelo método estatístico. Este foi o parâmetro que apresentou a menor concordância interobservador.

\section{Tomografia computadorizada}

Os dois radiologistas, ao analisarem os exames de TCSC, obtiveram as mesmas conclusões diagnósticas em 23 (92\%) dos 25 pacientes. Isto demonstra elevada concordância (Tabela 2).

\section{Urografia excretora}

Nos exames de UGE, apesar de não haver concordância em oito (32\%) dos 25 casos, esta não foi estatisticamente significante quando aplicamos o teste de McNemar (Tabela 2).

\section{TCSC $\times$ UGE}

Pudemos identificar o mesmo resultado entre os dois métodos (Figura 5) em $21(84 \%)$ dos 25 pacientes e discordân-

Tabela 1 Freqüência, em números absolutos, dos resultados da análise dos observadores A e $B$ dos exames de TCSC quanto ao aumento renal, hidronefrose, sinal do halo, estrias perirrenais e ureterais.

\begin{tabular}{|c|c|c|c|c|c|c|c|c|c|c|c|}
\hline & \multicolumn{10}{|c|}{ Observador A } \\
\hline & & \multicolumn{2}{|c|}{$\begin{array}{c}\text { Aumento } \\
\text { renal }\end{array}$} & \multicolumn{2}{|c|}{ Hidronefrose } & \multicolumn{2}{|c|}{ Sinal do halo } & \multicolumn{2}{|c|}{$\begin{array}{c}\text { Estrias } \\
\text { perirrenais }\end{array}$} & \multicolumn{2}{|c|}{$\begin{array}{c}\text { Estrias } \\
\text { periureterais }\end{array}$} \\
\hline & & + & - & + & - & + & - & + & - & + & - \\
\hline \multirow{2}{*}{ 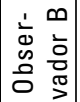 } & + & 20 & 0 & 19 & 3 & 18 & 1 & 18 & 1 & 13 & 3 \\
\hline & - & 1 & 4 & 3 & 0 & 6 & 0 & 4 & 2 & 5 & 4 \\
\hline
\end{tabular}

Tabela 2 Freqüência, em números absolutos, dos resultados da análise dos observadores $A$ e $B$, quanto à presença ou ausência de alterações urinárias vistas na TCSC e UGE.

\begin{tabular}{|c|c|c|c|c|c|}
\hline & \multicolumn{4}{|c|}{ Observador $\mathrm{A}$} \\
\hline & & \multicolumn{2}{|c|}{ TCSC } & \multicolumn{2}{|c|}{ UGE } \\
\hline & & + & - & + & - \\
\hline \multirow{2}{*}{$\begin{array}{l}\dot{1} \\
\dot{0} \\
\stackrel{0}{0} \\
\stackrel{0}{0} \\
\end{array}$} & + & 20 & 2 & 12 & 4 \\
\hline & - & 0 & 3 & 4 & 5 \\
\hline
\end{tabular}

cia em apenas quatro (16\%) exames, o que demonstra a boa concordância dos métodos (Tabela 3).

Quanto aos sinais tomográficos, verificamos suas freqüências em 22 pacientes que apresentavam exames positivos. Os sinais mais freqüentemente identificados foram, em $77 \%$ dos exames, a presença de cálculo ureteral, e em 68\% dos exames, a hidronefrose (Tabela 4).
A associação de sinais radiológicos na TCSC foi freqüente. A presença de dois sinais foi observada em $13,6 \%$ dos pacientes, três sinais em $18,2 \%$, quatro sinais em $27,3 \%$ e cinco sinais em $22,7 \%$ dos casos (Tabela 5).

\section{DISCUSSÃO}

A uropatia obstrutiva por impactação de cálculo no ureter, mesmo que momentânea, acomete $12 \%$ da população em geral, pelo menos uma vez na vida ${ }^{(8)}$. Aproximadamente 50\% desses pacientes apresentam recorrência em dez anos ${ }^{(9)}$. $\mathrm{O}$ diagnóstico tardio dessa alteração pode levar a complicações que implicam até perda funcional definitiva do rim acometido $^{(1)}$. Por esses motivos, diferentes métodos de diagnóstico por imagem têm sido utilizados para a avaliação de pacientes com cólica nefrética. 


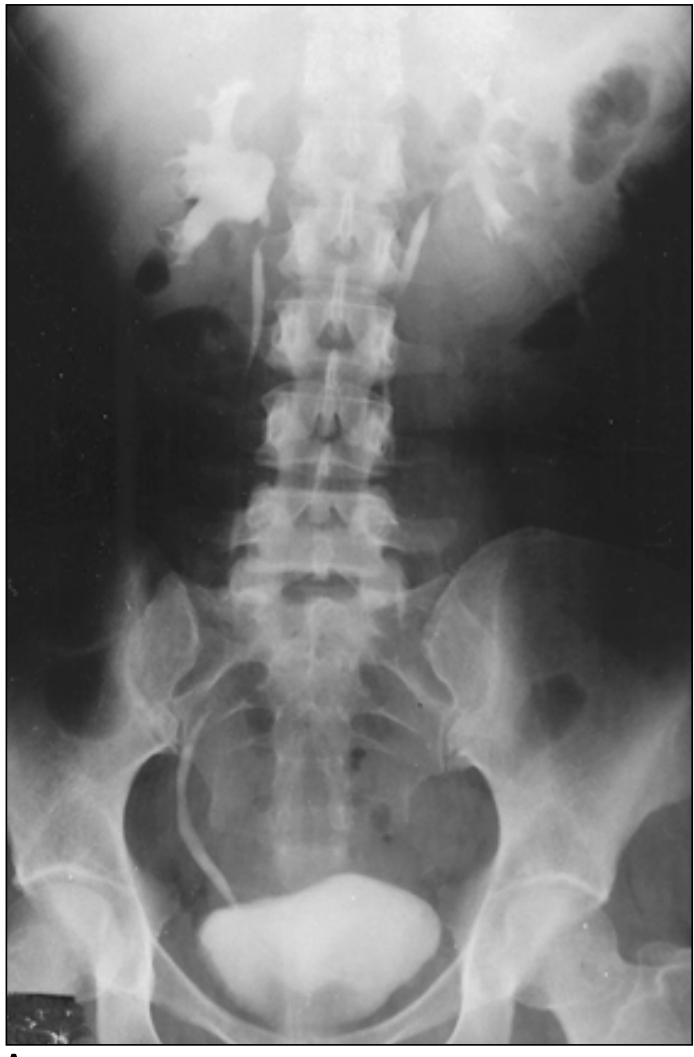

A

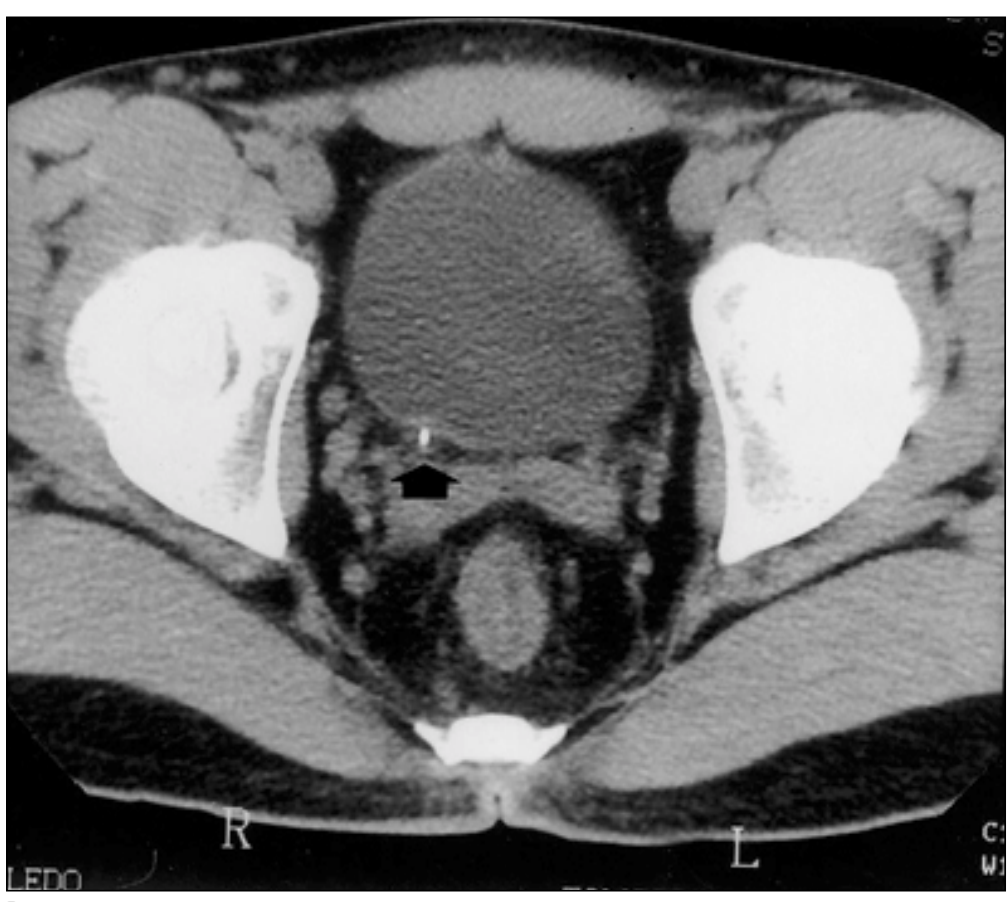

B

Figura 5. UGE (A) demonstrando ectasia da via urinária direita diagnosticada por este exame e também visibilizada à TCSC (B), que mostra cálculo na junção ureterovesical direita (seta) em paciente com cólica à direita.

Tabela 3 Freqüência, em números absolutos, da concordância entre os exames de TCSC e UGE, quanto à presença ou ausência de alterações urinárias.

\begin{tabular}{|c|c|c|c|c|}
\hline & \multicolumn{3}{|c|}{ Observador $A$} \\
\hline & & Positivo & Negativo & Total \\
\hline \multirow{3}{*}{ 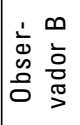 } & Positivo & 15 & 0 & 15 \\
\hline & Negativo & 4 & 6 & 10 \\
\hline & Total & 19 & 6 & 25 \\
\hline
\end{tabular}

Até um passado recente, a UGE era considerada o método mais apropriado para a avaliação dos pacientes com cólica nefrética, por suas elevadas sensibilidade $^{(\mathbf{1 0 , 1 1})}$ e especificidade ${ }^{(5)}$. Entre os limites da UGE podemos citar o uso de meio de contraste, tempo de exame, que pode se prolongar por várias horas devido a retardo na excreção renal, dificuldade na detecção de cálculos projetados sobre estruturas ósseas e limitações quanto a estabelecer um diagnóstico diferencial com a ureterolitíase.

O ultra-som, por ser um método simples, não-invasivo e amplamente disponível, teve ampla difusão, principalmen- te em pacientes com antecedentes alérgicos ao meio de contraste iodado. No entanto, limites técnicos do equipamento, características físicas do paciente, localização do cálculo e experiência do opea acurácia do método.

Estudos recentes procuraram demonstrar a eficácia da TCSC na avaliação de pacientes com cólica nefrética. Esses trabalhos apontam para uma sensibilidade que varia entre $96 \%$ e $98 \%$ e especificidade entre $98 \%$ e $100 \%^{(7,12,13)}$.

Assim como em outro estudo, demonstramos que a TCSC e a UGE são equivalentes no diagnóstico de ureterorador podem reduzir significativamente
Tabela 4 Freqüência, em porcentuais, dos sinais tomográficos estudados.

\begin{tabular}{|l|c|}
\hline \multicolumn{1}{|c|}{ Sinais } & Freqüência \\
\hline Aumento renal & $59 \%$ \\
Hidronefrose & $68 \%$ \\
Estrias perirrenais & $50 \%$ \\
Sinal do halo & $45 \%$ \\
Edema periureteral & $32 \%$ \\
Cálculo ureteral & $77 \%$ \\
\hline
\end{tabular}

Tabela 5 Freqüência, em porcentuais, da associação dos sinais tomográficos estudados nos exames de TCSC.

\begin{tabular}{|l|c|}
\hline \multicolumn{1}{|c|}{ Sinais associados } & Freqüência \\
\hline Dois & $13,6 \%$ \\
Três & $18,2 \%$ \\
Quatro & $27,2 \%$ \\
Cinco & $22,7 \%$ \\
\hline
\end{tabular}

litíase ${ }^{(13)}$. É importante salientar que a análise dos nossos exames foi realizada por médicos radiologistas com experiências diferentes, o que não interferiu nos resultados, assim como no estudo retros- 
pectivo realizado por Freed et al. ${ }^{(14)}$. Quando comparamos a reprodutibilidade dos métodos, notamos que a TCSC apresenta maior concordância entre examinadores do que a UGE, apesar da sua experiência profissional (Tabela 3). As análises acima sugerem maior facilidade na avaliação dos exames de TCSC do que de UGE em pacientes com cólica nefrética, mesmo para radiologistas menos experientes. Além disso, é importante salientar que a TCSC pode demonstrar mais sinais correlatos à ureterolitíase, como, por exemplo, a identificação de nefrolitíase pela TCSC em 13 pacientes, dos quais apenas sete apresentavam este achado na UGE (Figura 6).

A TCSC apresenta outras vantagens importantes, como a possibilidade de obter outros diagnósticos diferenciais extra-urinários (por exemplo, diverticulite aguda, apendicite aguda, apendicite epiplóica, entre outras doenças que podem simular o quadro clínico de ureterolitía- se), ao contrário da $\mathrm{UGE}^{(13)}$. O curto tempo de exame, que geralmente não excede os dez minutos entre o posicionamento e a dispensa do paciente, pode ser considerada outra vantagem da TCSC.

Para obtermos o diagnóstico de uropatia obstrutiva baixa por cálculo, na TCSC utilizamos como principal sinal a identificação do cálculo ureteral e outros sinais indiretos como a dilatação do sistema coletor, o edema perirrenal e periureteral e a nefromegalia. No nosso trabalho identificamos ureterolitíase em $77 \%$ dos pacientes com UGE alterada. A elevada freqüência deste achado se deve, provavelmente, ao protocolo do exame de TCSC. Foram realizados cortes finos $(6,5 \mathrm{~mm}$ de espessura) e com interpolação de $50 \%$ nas reconstruções $(3 \mathrm{~mm})$.

O diagnóstico diferencial entre cálculos e pequenos flebólitos não é fácil quando analisamos a escavação pélvica, próximo aos vasos ilíacos e gonadais. Traubici et al. ${ }^{(\mathbf{1 5 )}}$ demonstraram que a hi- pertransparência central de um flebólito, ao raio-x, não se traduz por hipodensidade à TCSC, como seria de se esperar. Nestas situações deve-se procurar acompanhar o ureter desde a junção ureteropiélica até seus segmentos distais e identificar sinais tomográficos indiretos.

Para todos os sinais tomográficos mencionados houve concordância entre os observadores (Tabelas 1 a 5). Apesar das avaliações de alguns desses parâmetros terem sido subjetivas, o fato de discutirmos previamente os sinais e só existirem duas variáveis possíveis (existência ou ausência) para cada um deles provavelmente ajudou no elevado índice de concordância. Houve discordância, mas não significante, apenas para os achados de estrias periureterais. Acreditamos que a causa da discrepância dos resultados talvez tenha sido a não-homogeneização da janela utilizada para a documentação do exame, dificultando assim a identificação de tênues estrias mimetizadas na

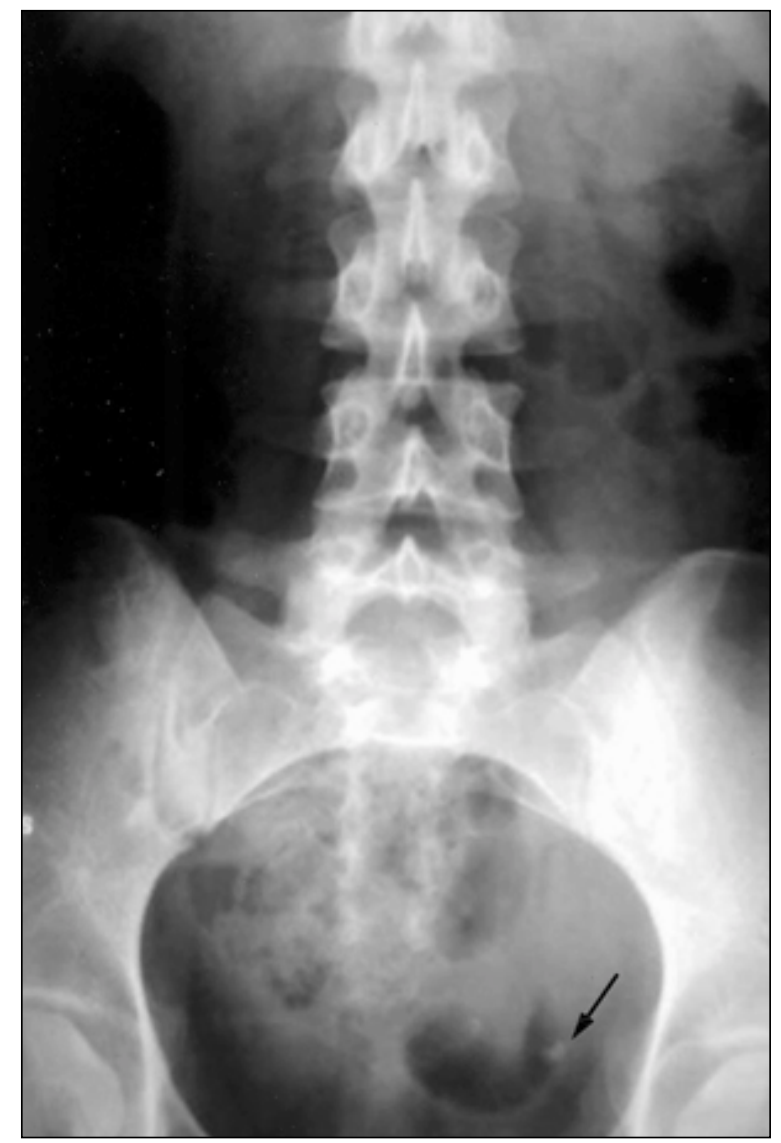

A

Figura 6. UGE (A), na sua radiografia simples já demonstrava ureterolitíase à esquerda (seta). A TCSC (B) identifica hidronefrose à esquerda (asterisco), causada pelo cálculo, e demonstra outros cálculos no rim direito (pontas de setas), não-visibilizados na UGE.

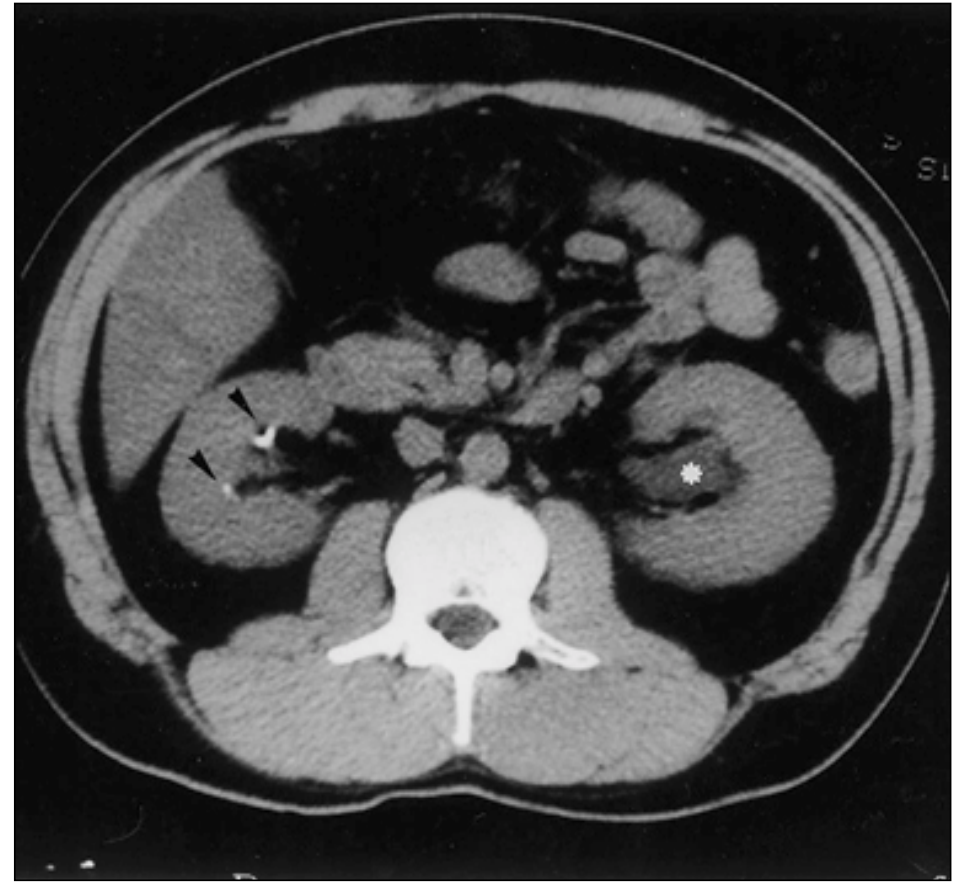

B 
gordura retroperitoneal. É importante salientar que estes sinais tomográficos indiretos não são específicos, mas quando combinados a outros e na presença de cólica nefrética são bastante eficientes na caracterização do quadro obstrutivo.

A diferenciação entre cálculo ureteral e flebólito é a principal causa de falha diagnóstica da TCSC, como mencionado anteriormente $^{(\mathbf{1 5})}$. Alguns sinais têm sido utilizados para distinguir estas condições, tais como o "sinal do halo", que consiste na identificação da parede do ureter em pacientes com cálculo, e o "sinal do cometa", correspondendo ao vaso na extremidade do flebólito. Na TCSC não é possível utilizar o sinal radiológico da hipertransparência central do flebólito, uma vez que foi demonstrada a baixa prevalência deste sinal nos exames tomográficos $^{(15)}$. As reconstruções coronais e parassagitais, associadas a criteriosa análise dos cortes axiais, pode auxiliar na diferenciação de cálculos e flebólitos.

Outro limite da TCSC reside na sua incapacidade em avaliar o grau de comprometimento da função renal, ao contrário da UGE. Apesar disso, alguns sinais tomográficos, como a presença de estrias perirrenais, aumento do volume renal e hipodensidade do parênquima, podem estar relacionados ao retardo da excreção renal identificado na UGE. Estudos posteriores poderão demonstrar a pertinência dessas observações.

Alguns urologistas sentem-se desconfortáveis em interpretar exames tomográ- ficos sem contraste, pela dificuldade em identificar as vias excretoras, principalmente quando não-dilatadas. O plano axial obtido nas imagens da TCSC é outra fonte de desconforto para aqueles especialistas acostumados apenas com as imagens panorâmicas e frontais da UGE. Por outro lado, temos notado crescente familiaridade e interesse por parte de alguns urologistas na utilização da TCSC em pacientes com suspeita de cálculo urinário. Este fato tem sido também relatado na literatura radiológica ${ }^{(\mathbf{1 6})}$.

\section{CONCLUSÃO}

Concluindo, a TCSC apresenta eficácia superponível à UGE na avaliação de pacientes com cólica nefrética, é um método reprodutível e bem aceito pelos pacientes, em virtude da sua rapidez e agilidade e sem o desconforto inerente à utilização do uso do meio de contraste endovenoso. Por essas razões, acreditamos que a TCSC terá papel proeminente na pesquisa de ureterolitíase e seus principais diagnósticos diferenciais.

\section{REFERÊNCIAS}

1. Davidson AJ, Hartman DS, Choyke PL, Wagner BJ. Davidson's Radiology of the kidney and genitourinary tract. 3rd ed. Philadelphia: Saunders, 1999.

2. Schmucki O, Asper R. Clinical significance of stone analysis. Urol Int 1986;41:343-7.

3. Deuwes W, Jager N. Harnblasen divertikulsteine. ROFO Fortschr Geb Rontgenstr Nuklearmed 1986; 144:731-2.

4. Levine JA, Neitlich J, Verga M, Dalrymple N, Smith RC. Ureteral calculi in patients with flank pain: correlation of plain radiography with unenhanced helical CT. Radiology 1997;204:27-31.

5. Svedstrom E, Alanen A, Nurmi M. Radiologic diagnosis of renal colic: the role of plain films, excretory urography and sonography. Eur Radiol 1990;11:180-3

6. Dalrymple NC, Verga M, Anderson KR, et al. The value of unenhanced helical computerized tomography in the management of acute flank pain. J Urol 1998;159:735-40.

7. Smith RC, Verga M, McCarthy S, Rosenfield AT. Diagnosis of acute flank pain: value of unenhanced helical CT. AJR 1996;166:97-101.

8. Sierakowski R, Finlayson B, Landes RR, Finlayson $\mathrm{CD}$, Sierakowski N. The frequency of urolithiasis in hospital discharge diagnoses in the United States. Invest Urol 1978;15:438-41.

9. Leusmann DB, Niggemann H, Roth S, von Ahlen H. Recurrence rates and severity of urinary calculi. Scand J Urol Nephrol 1995;29:279-83.

10. Mutgi A, Williams JW, Nettleman M. Renal colic: utility of the plain abdominal roentgenogram. Arch Intern Med 1991;151:1589-92.

11. Saita H, Matsukawa M, Fukushima H, Ohyama C, Nagata Y. Ultrasound diagnosis of ureteral stones: its usefulness with subsequent excretory urography. J Urol 1988;140:28-31.

12. Fielding JR, Steele G, Fox LA, Heller H, Loughlin KR. Spiral computerized tomography in the evaluation of acute flank pain: a replacement for excretory urography. J Urol 1997;157:2071-3.

13. Miller OF III, Rinner SK, Reichard SR, et al. Prospective comparison of unenhanced spiral computed tomography and intravenous urogram in the evaluation of acute flank pain. Urology 1998;52:982-7.

14. Freed KS, Paulson EK, Frederick MG, et al. Interobserver variability in the interpretation of unenhanced helical CT for the diagnosis of ureteral stone disease. J Comput Assist Tomogr 1998;22: 732-7.

15. Traubici J, Neitlich JD, Smith RC. Distinguishing pelvic phleboliths from distal ureteral stones on routine unenhanced helical CT: is there a radiolucent center? AJR 1999;172:13-7.

16. Preminger GM, Vieweg J, Leder RA, Nelson RC. Urolithiasis: detection and management with unenhanced spiral CT - a urologic perspective. Radiology 1998;207:308-9. 